Géographie physique et Quaternaire

\title{
Présence d'un pergélisol würmien sur le piémont des Pyrénées Atlantiques
}

Permafrost Structures of Würmian Age on the Northern

Piedmont of the Pyrénées

\section{Ein Permafrostboden aus der Würmeiszeit an der} Bergfussebene des atlantischen Pyrenäen

Pierre Gangloff, Bernard Hétu, François Courchesne et Pierre J. H. Richard

Volume 48, numéro 2, 1994

URI : https://id.erudit.org/iderudit/032994ar

DOI : https://doi.org/10.7202/032994ar

Aller au sommaire du numéro

\section{Éditeur(s)}

Les Presses de l'Université de Montréal

\section{ISSN}

0705-7199 (imprimé)

1492-143X (numérique)

Découvrir la revue

\section{Citer cet article}

Gangloff, P., Hétu, B., Courchesne, F. \& Richard, P. J. H. (1994). Présence d’un pergélisol würmien sur le piémont des Pyrénées Atlantiques. Géographie physique et Quaternaire, 48(2), 169-178. https://doi.org/10.7202/032994ar
Résumé de l'article

Des cryoturbations se sont produites sur le sommet de terrasses anciennes sur le piémont nord des Pyrénées. Elles déforment le contact entre une couche de loess wùrmien et les cailloutis altérés sous-jacents. Deux fentes de gel fossiles, sous la zone cryoturbée, indiquent la présence, sous la couche active, d'un pergélisol à plus de $15 \mathrm{~km}$ au nord des moraines externes de Lourdes. 


\section{PRÉSENCE D'UN PERGÉLISOL WÜRMIEN SUR LE PIÉMONT DES PYRÉNÉES ATLANTIQUES}

Pierre GANGLOFF', Bernard HÉTU ${ }^{2}$, François COURCHESNE ${ }^{1}$ et Pierre J.H. RICHARD ${ }^{1}$ : ${ }^{1}$ Département de Géographie, Université de Montréal, C.P. 6128, Succ. Centre-ville, Montréal, Québec H3C $3 \mathrm{J7} ;{ }^{2}$ Module de Géographie, Université du Québec à Rimouski, 300, allée des Ursulines, Rimouski, Québec G5L 3A1.

RÉSUMÉ Des cryoturbations se sont produites sur le sommet de terrasses anciennes sur le piémont nord des Pyrénées. Elles déforment le contact entre une couche de lœss würmien et les cailloutis altérés sousjacents. Deux fentes de gel fossiles, sous la zone cryoturbée, indiquent la présence, sous la couche active, d'un pergélisol à plus de $15 \mathrm{~km}$ au nord des moraines externes de Lourdes.

\begin{abstract}
Permafrost structures of Würmian age on the northern piedmont of the Pyrénées. Cryoturbations developed on top of old terraces of the northern piedmont of the Pyrénées. They deformed the contact between a Würmian loess and weathered gravels underneath. Two fossil ice wedge casts observed under the cryoturbated layer are indicative of a permafrost of Würmian age, extending more than $15 \mathrm{~km}$ north of the external moraines of Lourdes.
\end{abstract}

ZUSAMMENFASSUNG Ein Permafrostboden aus der Würmeiszeit an der Bergfussebene der atlantischen Pyrenäen. Kryoturbationen haben an der Oberfläche der alten Terrassen der Bergfussebene der Pyrenäen stattgefunden. Sie haben den Kontakt zwischen einer Löss-Schicht aus der Würmeiszeit und dem darunter liegenden verwitterten Geröll verformt. Zwei fossile Frost-Klüfte unter der kryoturbaten Zone weisen auf das Vorhandensein eines Permafrostbodens unter der aktiven Schicht, mehr als $15 \mathrm{~km}$ nördlich der externen Moränen von Lourdes. 


\section{INTRODUCTION}

Au Würm, un pergélisol continu couvrait en grande partie l'Europe occidentale. II s'étendait vers le sud jusqu'aux Alpes (fig. 1). Entre le Rhône et l'Atlantique, sa limite méridionale reste incertaine. Kaiser (1960) la situe dans les Pyrénées. Maarleveld (1976) et Velitchko (1982) la font s'arrêter à la latitude de Bordeaux où de nouvelles découvertes (Texier et Bertran, 1993) viennent confirmer son ancienne présence. II existe ainsi, au sud du Massif central, une zone d'incertitude d'environ 200 km (Pissart, 1987; Vandenberghe et Pissart, 1993) qu'une forte densité de gélistructures reliques, explicites, bien datées, permettraient de réduire. Nos observations se localisent dans cette zone, vers $43^{\circ}$ de latitude $\mathrm{N}$. Elles témoignent d'un pergélisol würmien à une quinzaine de kilomètres au-devant des moraines du piémont pyrénéen, une région où Poser, dès 1948, anticipait sa présence.

\section{LES DONNÉES STRATIGRAPHIQUES}

Entre Lourdes, Tarbes et Pau (fig. 2), des involutions se sont constituées sur le sommet des terrasses préwürmiennes. Elles déforment vigoureusement le contact entre une couche de limon recouvrant des alluvions profondément altérées. Deux coupes, à $6 \mathrm{~km}$ de distance (fig. 3 et tabl. I), ouvertes dans le cône de Ger (Formation de Lannemezan) et, $120 \mathrm{~m}$ en contrebas, dans la "terrasse moyenne ", en permettent l'étude détaillée. Un troisième site, près de Barzun (fig. 2, tabl. I) montre des guirlandes d'involution qui se suivent, dans un fossé de route, sur des dizaines de mètres de long. Faute d'une tranchée suffisamment profonde, ce site n'a pas été étudié. II n'en témoigne pas moins de l'abondance de ces structures sédimentaires et laisse présager de nouvelles découvertes.

SUR LE CÔNE DE GER : LA COUPE DE LUQUET (fig. 4)

Une tranchée de route montre de bas en haut:

- Le cailloutis de la Formation de Lannemezan, visible sur plus de 3,30 m d'épaisseur. Profondément altéré et rubéfié (Munsell 10 YR 7/8), ses galets et blocs ne dépassent pas $15 \mathrm{~cm}$ de grand axe. La plupart des roches sont décomposées en argile. Les quartzites cependant restent sains. Les cailloux baignent dans une matrice limono-argileuse, rubéfiée, renfermant des feuillets blancs d'halloysite (Icole, 1971, 1974 et communication personnelle). La formation est attribuée au Quaternaire ancien.

Sur les $0,70 \mathrm{~m}$ supérieurs, les fantômes de cailloux décomposés disparaissent.

Ce phénomène s'accompagne d'une forte proportion de galets sains redressés ${ }^{1}$ sur la tranche $(26 \%)$ ou sur la pointe (52\%). Un pavage de blocs quartzitiques coiffe le sommet de l'alluvion altérée. Les blocs du pavage ne sont pas jointifs et ne présentent pas d'orientation préférentielle. lls sont fossilisés par:

1. Sont considérés, ici, comme redressés, tous les galets et blocs dont l'un des axes a ou b est incliné à plus de $45^{\circ}$.
- Une couche de limon clair (2,5 YR 7/6) de 0,60 m à $1,10 \mathrm{~m}$ d'épaisseur. Elle renferme, à sa base, environ $30 \%$ d'argile, principalement de la kaolinite et du quartz avec, en moindre abondance, des micas et de la chlorite, mais très peu de feldspath (tabl. II et III).

Un régosol humique orthique ${ }^{2} s^{\prime} y$ est développé (tabl. IV). Sous $30 \mathrm{~cm}$ d'un horizon perturbé (AP) où s'entremèlent des matériaux issus de l'ensemble du profil, l'horizon Ah, brunâtre (10 YR 4/3), partiellement tronqué et enrichi en matière organique, pénètre sous forme de langues de $60 \mathrm{~cm}$ dans l'horizon $C$ sous-jacent. Au contact du cailloutis, l'horizon $C$ présente une frange gris foncée $(2,5$ YR $5 / 2)$ de $1 \mathrm{~cm}$ à $3 \mathrm{~cm}$ de large, enrichie en humus illuvié. L'absence d'horizon B témoigne d'une évolution pédologique peu poussée du profil.

L'horizon C est pollinifère. II renferme moins de 2600 grains par $\mathrm{cm}^{3}$, dont $88 \%$ d'herbes, principalement des Liguliflorae et seulement $8 \%$ d'arbres (tabl. V).

L'horizon Ah, par contre, contient jusqu'à 108000 grains de pollen par $\mathrm{cm}^{3}$. lls ne se répartissent pas de manière homogène, comme le montrent les écarts, du simple au double, entre deux échantillons d'un même horizon (tabl. V). Ici encore, les spectres polliniques se caractérisent par la prédominance des herbes, surtout des graminées sauvages (diamètre polaire des grains inférieur à $41 \mu \mathrm{m}$ ) et la présence du bouleau (9 à 11,5\%) et de l'aulne (9 à 10,5\%). II manque le pollen caractéristique des forêts, surtout de Pinus, et des défrichements holocènes décrits par Andrieu (1991), Jalut (1984) et Reille (1990). Les spectres évoquent la phase de Poaceae, du Würm supérieur, "que seules les séries de basse altitude des Pyrénées françaises ont enregistré, de la vallée de la Garonne ... à l'axe du Gave de Pau ... et d'Ossau. Dans le profil de Barbazan 1, cet événement débute à une date antérieure à $17650 \pm 330 \mathrm{yr} B P$ et se termine vers $15800 \pm 270$ yr BP " (Andrieu, 1991, p. 201).

Des galeries de vers de terre, de $6 \mathrm{~mm}$ à $10 \mathrm{~mm}$ de diamètre traversent le limon. Leur remplisage gris-brun, probablement coprogène, provient d'un mélange de l'horizon Ah et de l'horizon C. Ici encore, la concentration pollinique de deux échantillons présente des contrastes considérables (tabl. V). L'un d'eux comporte jusqu'à 733500 grains par $\mathrm{cm}^{3}$, dont $96,5 \%$ d'herbes, $1 \%$ d'aulnes et $1,7 \%$ de bouleaux aux grains généralement corrodés et dont le diamètre polaire, de $21 \mu \mathrm{m}$ en moyenne, indique des arbustes plutôt que des arbres. Les herbes, surtout des Liguliflorae et des graminées sauvages, se marquent par la rareté des cypéracées et la quasi absence d'Artemisia. Comme dans l'horizon Ah, les grains ou fragments de grains de Pinus, Picea, Quercus, lorsqu'ils sont présents, ne constituent qu'un bruit de fond.

Le dépôt de la couche de limon (horizon $\mathrm{C}$ ) puis sa pédogenèse (Ah) semblent s'être déroulés dans un milieu de steppe autorisant la croissance de l'aulne, peut-être en galeries le long des cours d'eau.

- Des involutions d'une amplitude de $50 \mathrm{~cm}$ déforment le contact entre limon et cailloutis de terrasse (fig. 4 et 5). La

2. Taxonomie et descriptions pédologiques selon le Comité d'experts sur la prospection pédologique d'Agriculture Canada, (1987). 


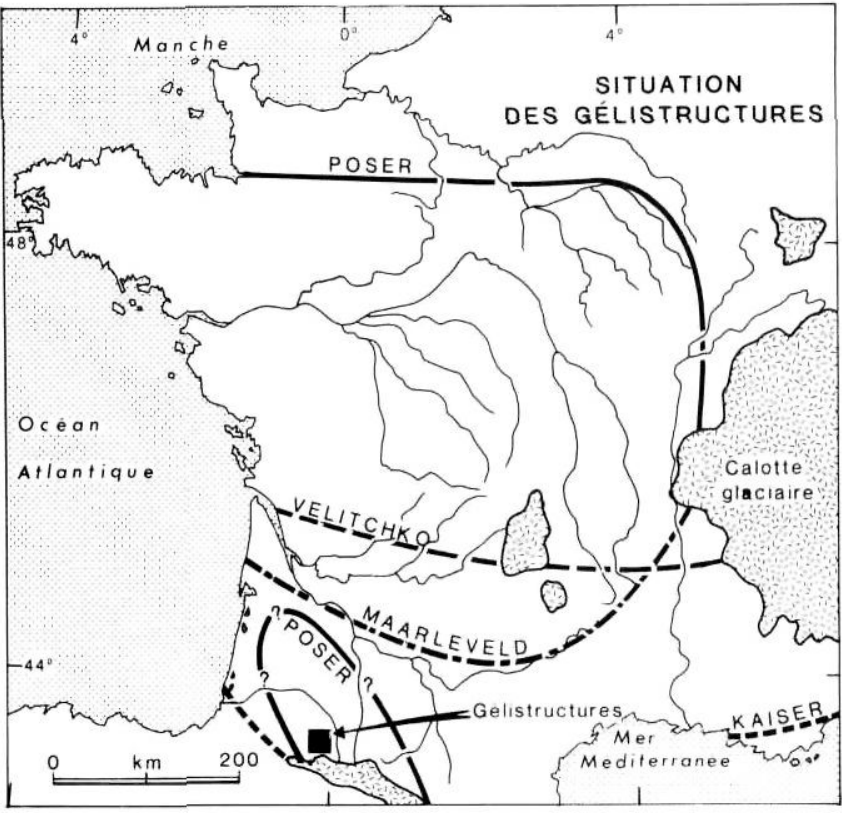

FIGURE 1. Localisation de la région étudiée par rapport aux limites méridionales du pergélisol würmien, selon différents auteurs. Redessinée d'après Pissart (1987, p. 122).

Location of studied area and southern limits of würmian permafrost, according to several authors. Redrawn from Pissart (1987, p. 122).

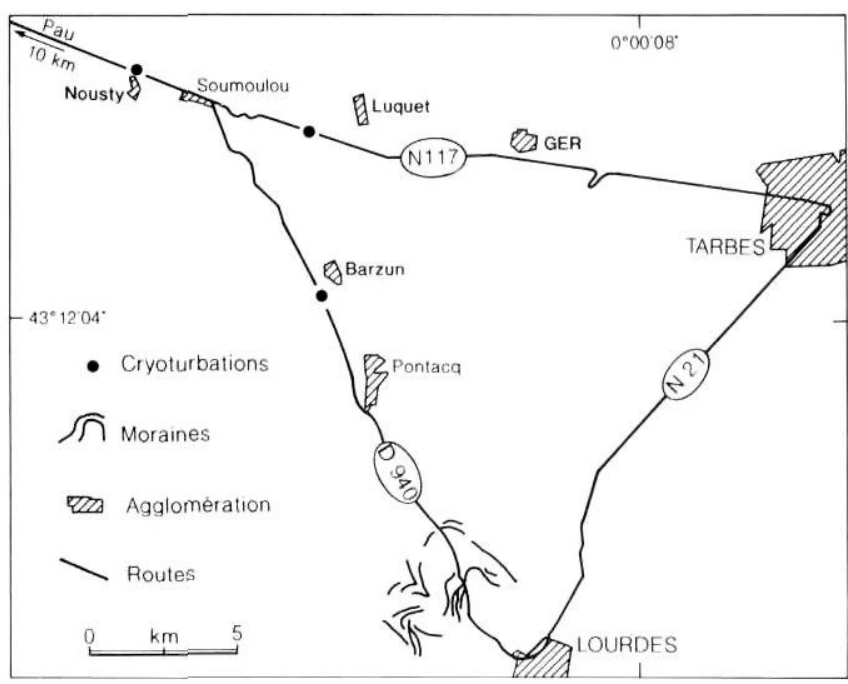

FIGURE 2. Localisation des sites à cryoturbations entre Lourdes, Tarbes et Pau.

Location of cryoturbated sites between Lourdes, Tarbes and Pau.

base de ces turbations, bien alignée, semble avoir été bloquée à $110 \mathrm{~cm}$ de profondeur. Deux fentes en coin étroites, remplies de limon brunâtre, amorphe, prolongent vers le bas deux poches d'involution. Larges de 2 à $3 \mathrm{~cm}$ à leur partie supérieure, elles descendent dans l'alluvion ancienne jusqu'à $160 \mathrm{~cm}$ et $180 \mathrm{~cm}$ de profondeur.

Dans l'ensemble, le pavage de blocs semble intact, les poches d'involution paraissant butter contre ses cailloux à peine dérangés. Au-dessus des fentes fossiles, toutefois, la
CRYOTURBATIONS:

contexte géomorphologique

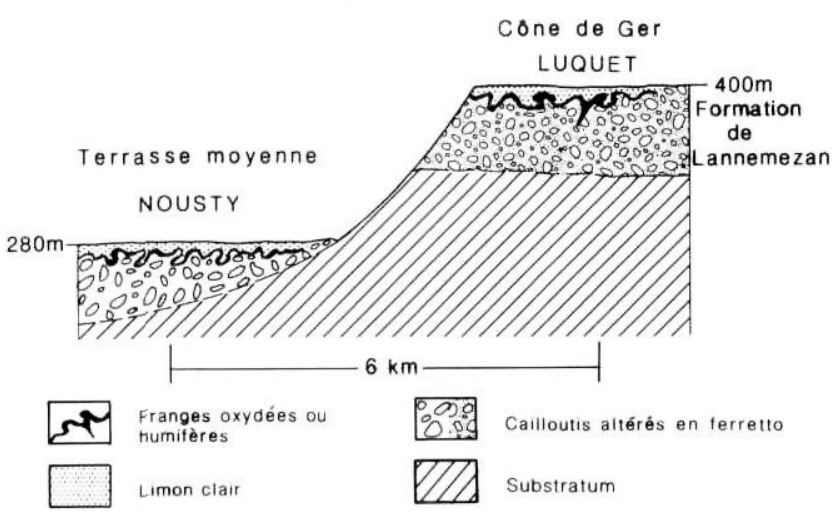

FIGURE 3. Étagement des sédiments cryoturbés.

Terrassing of cryotrubated sediments.

TABLEAU ।

Coordonnées des coupes

\begin{tabular}{lcccccc}
\hline & \multicolumn{3}{c}{ Localisation } & \multicolumn{3}{c}{ Zone cryoturbée } \\
\cline { 2 - 6 } Site & Long. & Lat. & Alt. & Sommet Amplitude & Base \\
\hline $\begin{array}{l}\text { Nousty } \\
\text { (N 117) }\end{array}$ & $0^{\circ} 12^{\prime} 36^{\prime \prime}$ & $43^{\circ} 16^{\prime} 30^{\prime \prime}$ & $280 \mathrm{~m}$ & $20 \mathrm{~cm}$ & $80 \mathrm{~cm}$ & $100 \mathrm{~cm}$ \\
$\begin{array}{l}\text { Luquet } \\
\text { (N 117) }\end{array}$ & $0^{\circ} 8^{\prime} 24^{\prime \prime}$ & $43^{\circ} 15^{\prime} 27^{\prime \prime}$ & $400 \mathrm{~m}$ & $60 \mathrm{~cm}$ & $50 \mathrm{~cm}$ & $110 \mathrm{~cm}$ \\
$\begin{array}{l}\text { Barzun } \\
\text { (D 940) }\end{array}$ & $0^{\circ} 8^{\prime} 20^{\prime \prime}$ & $43^{\circ} 12^{\prime} 07^{\prime \prime}$ & $350 \mathrm{~m}$ & & & $>100 \mathrm{~cm}$ \\
\hline
\end{tabular}

ligne de blocs est déformée. Certains cailloux, redressés, ont pénétré vers le haut dans la couche limoneuse. Un bloc, pris dans le limon, s'est affaissé vers la fente. Les galeries de vers de terre ne semblent pas dérangées par les involutions. Elles leur sont postérieures.

La chronologie suivante se dégage de la coupe. II y eut successivement :1) construction, au Quaternaire ancien, du cône de Ger; 2) altération de son matériel; 3) mise en place du pavage de blocs quartzitiques, les autres roches étant déjà décomposées; 4) dépôt, en milieu steppique, du limon sur le pavage de blocs; 5 ) développement des involutions et des fentes en coin; 6) pédogenèse dans un paysage de steppe et formation des galeries de vers de terre. Avant d'en tirer les enseignements paléoclimatiques, il est intéressant de comparer cette évolution avec celle de la "terrasse moyenne ", à $120 \mathrm{~m}$ en contrebas.

\section{SUR LA « TERRASSE MOYENNE » : LE SITE DE NOUSTY} (fig. 6)

La stratigraphie ne présente que peu de différences avec celle de Luquet. Une tranchée de route de 3,60 $\mathrm{m}$ de profondeur montre, de bas en haut :

- Le cailloutis de la terrasse, altéré, rubéfié, visible sur plus de $2,60 \mathrm{~m}$. Ses galets et blocs grossiers, roulés, atteignent $25-30 \mathrm{~cm}$ de grand axe. Ils baignent dans une matrice 


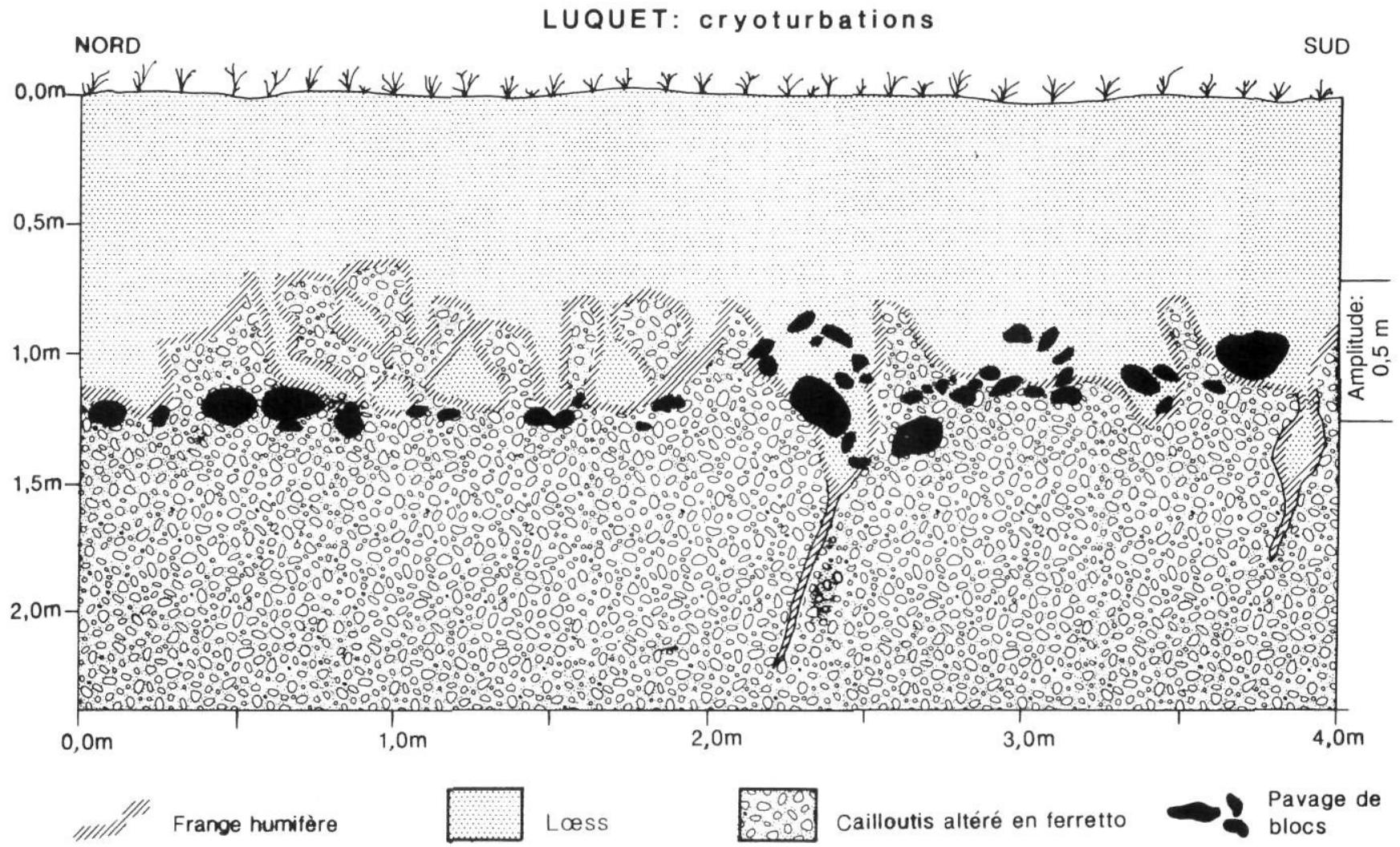

FIGURE 4. Coupe de Luquet montrant les cryoturbations au contact du lœss et du cailloutis de la Formation de Lannemezan (Pleistocène inférieur). Le pavage de blocs, indemne dans la partie gauche de la figure, est déformé dans la partie droite, avec pénétration de cailloux dans le lœss, au droit de fentes de gel fossiles.
Roadcut at Luquet exhibiting cryoturbations of the loess and alluvial deposits of the Formation de Lannemezan (lower Pleistocene). The stone line has been disturbed on the right part of the figure, where stones penetrated into the loess, above fossil ice wedge casts.

TABLEAU ॥

Granulométrie des coupes de Luquet, de Nousty et de Sesques

\begin{tabular}{|c|c|c|c|c|c|c|c|c|}
\hline & $\begin{array}{l}\text { Sable } \\
\text { t. gros }\end{array}$ & $\begin{array}{l}\text { Sable } \\
\text { gros }\end{array}$ & $\begin{array}{l}\text { Sable } \\
\text { moyen }\end{array}$ & $\begin{array}{c}\text { Sable } \\
\text { fin }\end{array}$ & $\begin{array}{l}\text { Sable } \\
\text { très fin }\end{array}$ & $\begin{array}{l}\text { Limon } \\
\text { gros }\end{array}$ & $\begin{array}{l}\text { Limon } \\
\text { fin }\end{array}$ & Argile \\
\hline & $2-1$ & $1-0,5$ & $0,5-0,25$ & $0,25-0,1$ & $0,1-0,053$ & $53-20$ & $20-2$ & $<2$ \\
\hline LUQUET & & & $\%$ & & & & & \\
\hline $\mathrm{Ah}$ & 0,9 & 1,9 & 3,9 & 5,9 & 3,4 & 20 & 48 & 16 \\
\hline II Cy & 1,0 & 1,7 & 3,3 & 5,2 & 3,8 & 17 & 37 & 31 \\
\hline \multicolumn{9}{|l|}{ NOUSTY } \\
\hline Ap & 1,4 & 2,4 & 5,0 & 6,3 & 4,9 & 27 & 42 & 11 \\
\hline Bgy1 & 1,0 & 1,1 & 1,6 & 2,2 & 2,1 & 22 & 41 & 29 \\
\hline Bgy2 & 1,6 & 1,4 & 1,6 & 2,1 & 2,3 & 16 & 37 & 38 \\
\hline \multicolumn{9}{|c|}{ SESQUES } \\
\hline S1 & 0 & 0 & 0 & 0 & 14 & 20 & 57 & 9 \\
\hline $\mathrm{S} 2$ & 0 & 0 & 0 & 0 & 14 & 27 & 49 & 10 \\
\hline
\end{tabular}

altérée. Parmi les blocs, les granites, météorisés, présentent souvent un cortex de $5 \mathrm{~mm}$ de large. Certains s'effritent entre les doigts. D'autres conservent une bonne cohérence. Les quartzites restent parfaitement sains. Au total, malgré une rubéfaction analogue à celle de Luquet, le degré d'altération de la terrasse moyenne est moins poussé, comme l'ont bien établi les études antérieures (Alimen, 1964; Icole, 1974). La "terrasse moyenne " remonte à l'avant dernière glaciation (Hubschmann et al., 1980).

Dans les $80 \mathrm{~cm}$ supérieurs, les galets, moins abondants au sein d'une abondante matrice argilo-limoneuse, sont 
TABLEAU III

Minéralogie de la fraction argileuse des coupes de Luquet, de Nousty et de Sesques

\begin{tabular}{lccccccc}
\hline Échantillon & Chl. & Int. & Mi. & Kao. & Qz. & Feld. & Am. \\
\hline Luquet Ah & 2 & 1 & 2 & 3 & 4 & 1 & - \\
Luquet IICy & 2 & 1 & 2 & 4 & 3 & 1 & - \\
Nousty Bgy1 & 2 & 1 & 2 & 3 & 4 & 1 & - \\
Nousty Bgy2 & 1 & 1 & 3 & 3 & 4 & 1 & - \\
Nousty IICy & 1 & 1 & 3 & 3 & 4 & 1 & - \\
Sesqès & 3 & - & 3 & - & 4 & 1 & 2 \\
\hline
\end{tabular}

$4:$ très abondant; $3:$ majeur; $2:$ mineur; $1:$ trace.

Chl. : Chlorite; Int. : Insterstratifiés; Mi. : Mica; Kao. : Kaolinite; Qz. : Quartz; Feld. : Feldspath; Am. : Amphibole.

redressés soit sur la tranche $(21 \%)$, soit sur la pointe $(47 \%)$. On y observe, vers le haut, une structure lamellaire, centimétrique et des blocs limono-argileux polyédriques. Ce type de structure est parfois associé au développement d'un horizon fragique, bien qu'il ne s'agisse pas ici d'un fragipan, au sens pédologique du terme. La désagrégation rapide des blocs de limon dans l'eau l'exclut.

- Une couche de limon clair, de 0,20 m à $1 \mathrm{~m}$ d'épaisseur, repose sur l'alluvion. Aucune nappe de gravats ne sépare les deux formations. Par sa couleur, sa texture marquée par un enrichissement en argile vers le bas de la couche (tabl. II), son faible degré de pédogenèse (tabl. VI) et la minéralogie de sa fraction argileuse (tabl. III), le limon ressemble à celui de Luquet. II renferme, à mi-hauteur, une bande humifère, peutêtre un paléosol embryonnaire. Un gleysol humique orthique (tabl. VI) s'est développé dans l'ensemble du faciès. II comporte un Ap d'environ $20 \mathrm{~cm}$ surmontant des horizons B (Bgy) riches en marbrures (10 YR 6/8) attestant des cycles d'oxydation et de réduction. La gleyification ne constitue cependant pas le processus pédogénétique actuel. En effet, des accumulations de matière organique illuviale le long des réseaux poreux et racinaires recoupent le profil. La translocation de la matière organique est moins prononcée que dans le profil de Luquet.

La couche de limon ne comporte pas de tunnels de vers de terre. D'un point de vue palynologique, elle est stérile. A sa base, une frange centimétrique d'oxyde de fer souligne le contact avec le cailloutis.

- Des involutions, ici encore, déforment le contact entre limon et cailloux. Comme à Luquet, la base des poches limoneuses s'arrête à une profondeur uniforme, ici vers $1 \mathrm{~m}$. Entre les poches, le cailloutis altéré s'élève jusqu'à $20 \mathrm{~cm}$ de la surface. Le contact sinusoïdal entre les deux faciès présente une longueur d'onde de l'ordre de $20 \mathrm{~cm}$ à $30 \mathrm{~cm}$ et une amplitude de $80 \mathrm{~cm}$. Le niveau humifère à l'intérieur du limon a été plissé et localement laminé.

Vers $1 \mathrm{~m}$ de profondeur, des taches rondes de limon percent dans le cailloutis (fig. 6). Elles indiquent l'affleurement, dans la paroi de la coupe, de langues d'involution présentes en retrait, dans le matériel alluvial. L'une de ces
TABLEAU IV

Profil pédologique de Luquet

\begin{tabular}{|c|c|c|c|c|c|}
\hline Horizon & $\begin{array}{l}\text { Profondeur } \\
\mathrm{cm}\end{array}$ & Couleur & Texture & ${ }^{a}$ Structure ${ }^{b}$ & Remarques \\
\hline Ap & $0-30$ & 10 YR $4 / 3$ & LLi & 2-m-pos & Perturbé \\
\hline $\mathrm{Ah}$ & $30-40$ & $2,5 Y \quad 4 / 2$ & LLi & 2-m-pos & \\
\hline$A$ et $C$ & $40-96$ & $2,5 Y \quad 6 / 6$ & LLi & 2-g-pos & Interpénétration $\mathrm{A}$ et $\mathrm{C}$ \\
\hline IICy & $96+$ & 7,5 YR $4 / 6$ & LLIA & $0-\mathrm{ma}$ & $\begin{array}{l}\text { Pavage de blocs, } \\
\text { turbations }\end{array}$ \\
\hline
\end{tabular}

a LLi : Lœm limoneux; LLiA : Lœam limono-argileux

b 2-m-pos Peds bien formés, moyens et polyédriques subanguleux;

2-g-pos Peds bien formés, gros et polyédriques subanguleux;

0-ma Sans structure, massif.

Lœam est pris au sens de Birkeland (1984) et du Comité d'experts sur la prospection pédologique d'Agriculture Canada (1987).

poches contient des charbons de bois millimétriques datés au radiocarbone de $2940 \pm 80 \mathrm{BP}$ (Beta-54611 et CAMS-3227). Une fissure millimétrique, tapissée de limon gris, accolée à une poche d'involution, descend dans le matériel de terrasse jusqu'à $1,75 \mathrm{~m}$ de profondeur.

\section{INTERPRÉTATION}

Ces deux coupes posent le problème de la nature et de l'âge des limons superficiels, de la genèse des structures sédimentaires et de leur signification paléogéographique.

\section{LA NATURE ET L'ÂGE DES LIMONS SUPERFICIELS}

Sommes-nous en présence de limons de débordement?

Leur granulométrie semble indiquer une origine fluviatile. L'abondance d'argile et la présence de sable au sein d'un matériel essentiellement limoneux évoque un dépôt par décantation, à la fin de crues épisodiques, par des cours d'eau faiblement encaissés dans les deux nappes alluviales caillouteuses.

Cette interprétation se heurte néanmoins à des difficultés d'ordre chronologique. À Luquet comme à Nousty, les limons n'ont subi qu'une faible pédogenèse. Ils reposent sur des alluvions rubéfiées et profondément décomposées par l'altération.

Rien ne s'oppose, en principe, à ce qu'au site de Luquet, une couche de limon alluvial se soit déposée sur une terrasse ancienne, déjà altérée. Mais, par la suite, ce limon frais aurait été exposé à la météorisation pendant que, $120 \mathrm{~m}$ en contrebas, la " terrasse moyenne " de Nousty se construit puis s'altère. Comment cette phase d'altération aurait-elle, à Nousty, rubéfié et décomposé des galets sur plus de $2,60 \mathrm{~m}$ de profondeur sans atteindre le limon de Luquet, à seulement $6 \mathrm{~km}$ de là? Lorsque la terrasse de Nousty subit son altération profonde, le limon de Luquet ne s'est pas encore déposé. II est nécessairement postérieur à la construction de la " terrasse moyenne " et à sa météorisation ultérieure.

Dès lors, il ne saurait être d'origine fluviatile. Au moment de son dépôt, les cours d'eau de la région s'écoulaient $120 \mathrm{~m}$ en contrebas du site. Aucune crue, si forte soit-elle, ne peut 


\section{TABLEAU $V$}

Palynologie du lœss de Luquet et du site de Sesques et spectre récent enregistré dans la région

\begin{tabular}{|c|c|c|c|c|c|c|c|c|c|c|}
\hline \multirow[b]{2}{*}{$\begin{array}{c}\text { Concentration } \\
\text { x1000 grains } / \mathrm{cm}^{3} \text { : }\end{array}$} & \multicolumn{2}{|c|}{ Horizon Ah } & \multicolumn{2}{|c|}{ Galeries de vers } & \multicolumn{2}{|c|}{ Horizon $\mathrm{C}$} & \multicolumn{2}{|c|}{ Sesques } & \multicolumn{2}{|c|}{ Spectres récents } \\
\hline & 47,9 & 108,4 & 57,6 & 733,5 & 1,3 & 2,6 & & & & \\
\hline ARBRES & $17,8 \%$ & $11,2 \%$ & $23,8 \%$ & $2,0 \%$ & $11,8 \%$ & $9,1 \%$ & $30,9 \%$ & $94,1 \%$ & $\begin{array}{l}\min . \\
28 \%\end{array}$ & $\begin{array}{l}\max . \\
100 \%\end{array}$ \\
\hline Abies & 0,5 & - & 0,3 & - & & & 1,2 & 10,3 & 0 & 10 \\
\hline Acer & & & 0,3 & - & & & & & 0 & $<1$ \\
\hline Betula & 11,4 & 9,2 & 20,2 & 1,7 & 2,9 & 4,5 & & 0,8 & 0 & 8 \\
\hline Picea & & & & & 4,4 & - & 0,4 & 0,2 & 0 & $<1$ \\
\hline Pinus & 2,5 & 0,6 & 0,6 & 0,1 & 3,0 & 2,0 & 19,5 & 81,4 & 25 & 85 \\
\hline Larix & & & 0,3 & - & & & & & 0 & 0 \\
\hline Quercus & 2,4 & 1,8 & 1,8 & 0,2 & 1,5 & 0,3 & 4,7 & 1,0 & 3 & 10 \\
\hline Fagus & & & 0,3 & - & & & 3,9 & 0,2 & 0 & 10 \\
\hline Tilia & 1,0 & 0,6 & & & & & & & 0 & 1 \\
\hline Ulmus & & & & & - & 0,3 & 0,4 & & 0 & 5 \\
\hline ARBUSTES & $9,3 \%$ & $10,6 \%$ & $17,9 \%$ & $1,0 \%$ & $7,4 \%$ & $1,2 \%$ & $2,4 \%$ & $0,9 \%$ & $0 \%$ & $>25 \%$ \\
\hline Alnus & 8,8 & 10,6 & 17,9 & 1,0 & 1,5 & 1,2 & 0,8 & 0,7 & 0 & 2 \\
\hline Myricaceae & & & & & 1,5 & - & 0,4 & & 0 & $<1$ \\
\hline Ericaceae & 0,5 & - & & & 4,4 & - & & 0,2 & 0 & 20 \\
\hline HERBES & $43,5 \%$ & $74,8 \%$ & $56,9 \%$ & $96,5 \%$ & $78,0 \%$ & $87,5 \%$ & $62,9 \%$ & $4,2 \%$ & $4 \%$ & $>87 \%$ \\
\hline Cyperaceae & 8,3 & 9,5 & 2,1 & 2,5 & 5,9 & 3,1 & 0,4 & & 4 & 25 \\
\hline Poaceae & 20,0 & 56,5 & 33,3 & 9,7 & 1,5 & 6,6 & 53,3 & 0,8 & 0 & 45 \\
\hline Liguliflorae & 0,9 & 1,5 & 18,3 & 83,4 & 54,4 & 69,3 & 0,4 & 1,0 & 0 & 8 \\
\hline Artemisia & 3,4 & 0,9 & 0,7 & 0,1 & 7,3 & 0,3 & 0,4 & 1,0 & 0 & $<1$ \\
\hline type Ambrosia & 1,0 & 1,2 & 1,1 & - & 1,5 & 2,1 & & & 0 & 0 \\
\hline Tubuliflorae & 1,0 & 1,8 & 0,3 & 0,7 & - & 3,1 & & 0,5 & & 1 \\
\hline Fabaceae & 0,5 & - & 1,1 & 0,1 & - & 0,7 & & & 0 & 7 \\
\hline Ranunculaceae & 1,5 & 0,6 & & & 2,9 & 1,0 & 0,4 & 0,2 & 0 & $<1$ \\
\hline Caryophyllaceae & 0,5 & 0,3 & & & 1,5 & 0,7 & 1,5 & & 0 & $<1$ \\
\hline Brassicaceae & 0,5 & 0,6 & & & 1,5 & 0,3 & 0,4 & 0,5 & 0 & $<1$ \\
\hline Chenopodiaceae & 0,5 & - & & & - & 0,3 & 1,9 & 0,2 & 0 & $<1$ \\
\hline cf. Scabiosa & 4,9 & 0,9 & & & & & & & 0 & $<1$ \\
\hline Rosaceae & 0,5 & - & & & & & & & 0 & $<1$ \\
\hline Campanulaceae & & & & & 1,5 & - & & & 0 & $<1$ \\
\hline Polygonum viviparum & & & & & - & 0,7 & & & 0 & 0 \\
\hline AUTRES & $29,3 \%$ & $3,6 \%$ & $1,4 \%$ & $0,5 \%$ & $2,9 \%$ & $2,1 \%$ & $4,0 \%$ & $0,8 \%$ & . & . \\
\hline Spore trilète (exclues) & & & & & - & $(0,7)$ & & & 0 & (24) \\
\hline Inconnus & 9,3 & 0,6 & & & & & & & 0 & 0 \\
\hline Indéterminables & 20,0 & 3,0 & 1,1 & 0,2 & 2,9 & 2,1 & 3,8 & 0,8 & 0 & 0 \\
\hline SOMME POLLINIQUE & 205 & 338 & 279 & 797 & 68 & 287 & 257 & 389 & . & . \\
\hline
\end{tabular}

Deux échantillons distincts furent traités et analysés pour chaque entité du site de Luquet. Ils permettent d'évaluer la variabilité interne de leur contenu pollinique. Les disparités les plus flagrantes sont identifiées par les rectangles, les autres, par des tirets. Quant au "spectre récent", la gamme (min. - max.) des pourcentages polliniques est tirée des travaux d'Andrieu (1991), de Jalut (1984) et de Reille (1990) pour le Sub-Atlantique. 


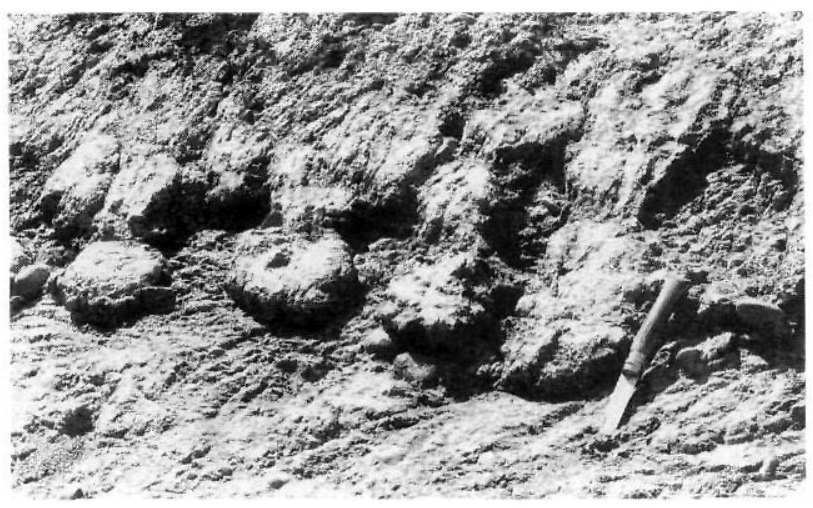

FIGURE 5. Photographie des cryoturbations de Luquet. Photograph of the Luquet cryoturbations.

être invoquée pour expliquer sa mise en place sur le cône de Ger.

\section{Le limon est d'origine éolienne}

À Luquet comme à Nousty, la granulométrie, la minéralogie de la fraction argileuse et le faible degré de pédogenèse montrent que nous sommes en présence d'un seul et même faciès. Parce qu'il recouvre des niveaux topographiques étagés et des unités géomorphologiques d'âges variables, il ne peut s'agir que d'un sédiment éolien, en d'autres termes, un lœss. Postérieur à la "terrasse moyenne ", attribuée à l'avant-dernière glaciation, ce lœss date ainsi du Würm.

Sa texture ne contredit-elle pas cette conclusion? Certes ses courbes granulométriques ne correspondent pas à celles des lœss classiques. Mais elles présentent une parenté indiscutable avec les courbes granulométriques d'un dépôt nivéoéolien actuel, prélevé sur une congère de neige, au fond du cirque de Sesques, dans les Pyrénées, vers $1870 \mathrm{~m}$ d'altitude, en pleine prairie d'altitude, à $50 \mathrm{~km}$ seulement au sudouest des sites à cryoturbations (fig. 7).

La seule différence notable entre le lœss de LuquetNousty et le sédiment nivéo-éolien de Sesques s'observe à la fraction sableuse. À Sesques, le sable ne dépasse jamais $100 \mu \mathrm{m}$ alors que le lœss du piémont comprend des sables fins, moyens et grossiers. Mais cette différence s'explique par des artifices statistiques et par le contexte géomorphologique. Les faibles pourcentages de sable grossier n'ont pas de valeur statistique, quelques grains, par leur taille et donc par leur masse, pouvant facilement valoir $1 \%$ de l'échantillon. Ces grains isolés peuvent provenir d'incorporations tardives à partir du cailloutis inférieur, à l'instar de certains blocs de la nappe de gravats de Luquet, ou à partir du sommet à l'instar des charbons de bois dont il sera question plus loin. La présence de sable moyen implique un transport éolien sur de très courtes distances à partir des alluvions locales.

La minéralogie des argiles est instructive. Dans le nivéoéolien de Sesques comme dans le lœss des terrasses dominent les minéraux de quartz, de micas et de chlorite, les feldspaths étant peu présents. Le lœss des terrasses contient cependant de la kaolinite, absente à Sesques. Or les blocs de granites, dans les alluvions anciennes, sont décomposés

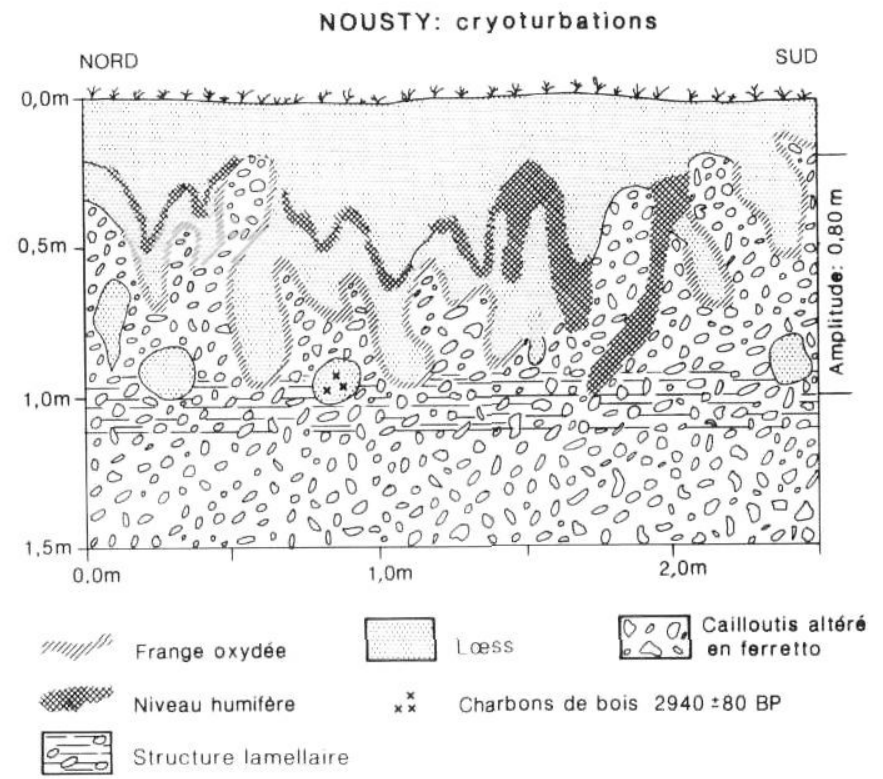

FIGURE 6. Coupe dans la terrasse moyenne à Nousty.

Roadcut entrenching the middle terrace at Nousty.

en kaolinite (Hétu et Gangloff, 1989). Ce minéral aurait donc été incorporé dans le lœss, à partir du matériel local.

C'est par la composition palynologique que les deux types de limons éoliens diffèrent radicalement. Ceux de Sesques, bien que déposés dans un milieu de prairie d'altitude, renferment jusqu'à $94 \%$ de grains d'arbres, dont $81 \%$ pour les seuls pins (tabl. V). Dans des mousses à côté de la congère de neige de Sesques, la proportion des arbres tombe à $31 \%$, alors que celle des herbes s'élève à $63 \%$. Ainsi, les mousses contiennent la pluie sporopollinique locale, alors que le limon nivéo-éolien voisin enregistre les apports polliniques régionaux, notamment des forêts en contrebas du site. Le lœss de Luquet et de Nousty, où domine le pollen d'herbes, pourrait lui-aussi correspondre à la végétation régionale, soit une steppe, contemporaine de son dépôt (horizon C), mais encore présente lors la pédogenèse subséquente (horizon Ah).

\section{L'ÂGE ET LA SIGNIFICATION DES INVOLUTIONS.}

Les involutions sont postérieures au lœss würmien qu'elles déforment. Elle datent donc, soit du Würm lui-même, soit de l'Holocène. Un âge holocène ne peut être rejeté d'emblée, en raison de la date de $2940 \pm 80$ BP obtenue sur le charbon de bois d'une poche d'involution. La date est-elle fausse? Probablement pas. Des feux sont attestés, vers $3050 \pm 100 \mathrm{BP}$, dans la vallée morte d'Ogeu, à $28 \mathrm{~km}$ au sudouest de Nousty. Fait troublant, à Ogeu, les charbons de bois recouvrent des involutions incontestablement holocènes, développées dans des lutites lacustres et attribuées à une dynamique de motturaux dans un marais (Gangloff et al., 1991a).

Faut-il dès lors envisager que toutes les turbations, y compris celles de Luquet et de Nousty, aient pu se former sous les conditions climatiques holocènes? Mais quel processus 
TABLEAU $V I$

Profil pédologique de Nousty

\begin{tabular}{|c|c|c|c|c|c|}
\hline Horizon & $\begin{array}{l}\text { Profondeur } \\
\mathrm{cm}\end{array}$ & Couleur & Texture & ${ }^{a}$ Structure ${ }^{b}$ & Remarques \\
\hline Ap & $0-22$ & $2,5 Y 7 / 4$ & LLi & 2-m-pos & Culture de maïs \\
\hline Bgy1 & $22-53$ & $2,5 Y 7 / 4$ & LLIA & 3-f-pos & Gleyification, turbations \\
\hline Bgy2 & $53-90$ & $2,5 Y 7 / 5$ & LLIA & 3-m-pos & Gleyification, turbations \\
\hline IICy & $90+$ & 2,5 Y $8 / 4$ & $\mathrm{LLi}$ & 0-ma & $\begin{array}{l}\text { Turbations-structure } \\
\text { lamellaire discontinue }\end{array}$ \\
\hline
\end{tabular}

a LLi : Lœm limoneux; LLiA : Lœm limono-argileux

b 2-m-pos Peds bien formés, moyens et polyédriques subanguleux;

3-f-pos Peds très bien formés, fins et polyédriques subanguleux;

3-m-pos Peds très bien formés, moyens et polyédriques subanguleux;

0-ma Sans structure, massif.

récent aurait déformé les cailloutis de terrasses jusqu'à $1,10 \mathrm{~m}$ de profondeur? Sans qu'on ne voit ce processus à l'œuvre de nos jours? De plus, comment expliquer, à Luquet, qu'après la formation d'involutions supposées postglaciaires, apparaissent des galeries de vers de terre renfermant des spectres polliniques de steppe? L'hypothèse de turbations holocènes ne tient pas. Le charbon de bois millimétrique a dû être introduit, peut-être par des animaux fouisseurs, dans le lœss, bien après la mise en place des involutions.

Ces involutions présentent l'allure typique des cryoturbations, plus précisément des Tropfenböden des auteurs allemands (Bülow, 1964; Eissmann, 1981). Sont-elles nécessairement périglaciaires? La seule gravité ne peut-elle les expliquer? Si des structures de charges, les load casts des auteurs anglophones, peuvent se produire par injection d'une couche dense recouvrant un faciès liquéfié (Washburn, 1973), on sait maintenant qu'en milieu sub-aérien, dans des cailloutis de terrasse, en l'absence de gel (Gullentops et Paulissen, 1978), un tel processus azonal est improbable. Exigeant des cycles gélivaux, ces structures sédimentaires sont d'authentiques cryoturbations. Aux considérations théoriques et aux expériences de laboratoire (Pissart,1982) privilégiant le rôle des pressions cryostatiques, Eissmann (1981, p. 23) ajoute un argument de poids en faveur d'une origine périglaciaire des Tropfenböden. Dans les exploitations de lignite, près de Leipzig, les fronts de coupes totalisant $100 \mathrm{~km}$ de long renferment des milliers de turbations dans leurs couches quaternaires et n'en contiennent pas une seule dans les faciès tertiaires similaires, sous le niveau atteint par le gel pléistocène.

Les involutions de Luquet et de Nousty impliquent donc des cycles gélivaux s'exerçant à plus d'un mètre de profondeur. Leur genèse exige, au minimum, des paléotempératures moyennes annuelles de l'air inférieures ou égales à $2^{\circ} \mathrm{C}$, avec des hivers rigoureux, dont la moyenne du mois le plus froid devait atteindre $-15^{\circ} \mathrm{C}$. Des cryoturbations se forment encore de nos jours sous de telles conditions, même sous forêt boréale, vers $48^{\circ}$ de latitude Nord (Brown et Gangloff, 1980). II s'agit là d'un seuil thermique supérieur impliquant, pour le piémont pyrénéen, un paléoclimat continental d'au moins $10^{\circ} \mathrm{C}$ plus froid que de nos jours. Comme les sites de Luquet et de Nousty ne se localisent qu'à 120 km de l'Atlantique, le caractère continental du paléoclimat pourrait corres-

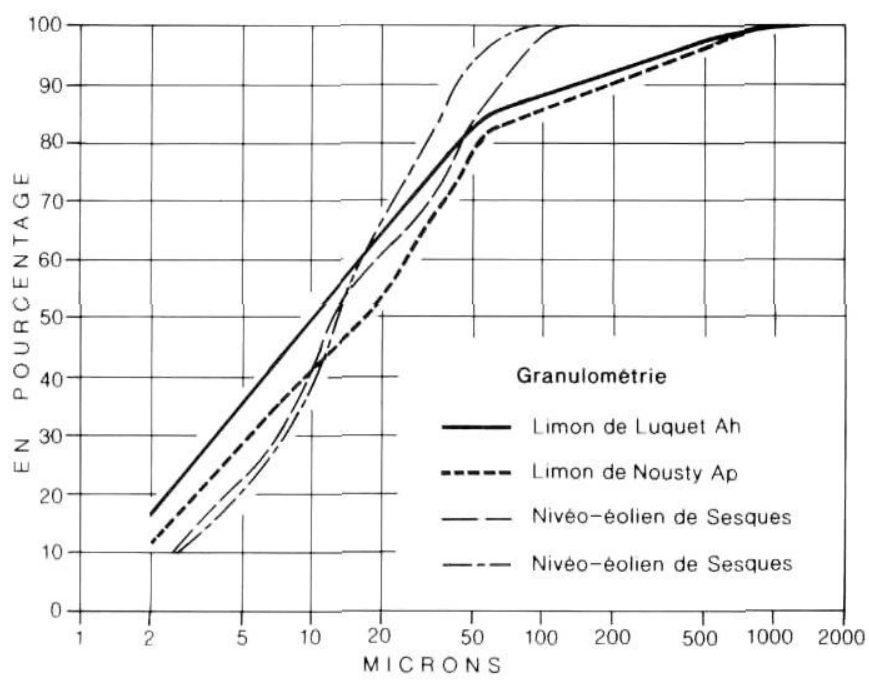

FIGURE 7. Comparaison des courbres granulométriques d'un sédiment nivéo-éolien actuel des Pyrénées et du lœss des terrasses de Nousty et Luquet. La seule différence notable réside dans la répartition des $15 \%$ à $18 \%$ de fraction sableuse.

Comparison between granulometric curves of present day niveoaeolian deposits in the Pyrénées and the loess of Luquet and Nousty.

pondre aux périodes würmiennes au cours desquelles la banquise couvrait l'océan.

Mais certains traits caractéristiques des gélistructures de Nousty et de Luquet impliquent des paléotempératures beaucoup plus basses que le seuil thermique supérieur évoqué ci-dessus. En effet, l'alignement de la base des involutions, la forme aplatie de plusieurs d'entre elles et le fait que ces structures en load casts pénètrent dans un matériel caillouteux, qui devait être sursaturé, implique, sous la couche cryoturbée, la présence d'un pergélisol (Bülow, 1964; Eissmann, 1981; Heyse, 1983; Vandenberghe et Van de Broek, 1982). La structure lamellaire observée sous les cryoturbations de Nousty plaide dans le même sens. Elle résulte probablement de lentilles de glace de ségrégation, comme on peut en observer encore de nos jours, par exemple dans les argiles de I'Ungava (Gangloff et Pissart, 1983; Pissart et Gangloff, 1984) en milieu de pergéliosl discontinu.

\section{LA SIGNIFICATION DES FENTES FOSSILES}

Ce pergélisol, les fentes en coin l'attestent. Certes des failles, des fissures de décollement sur des versants, des affaissements de culots de glace morte ou la dessiccation de matériel fin (Black, 1976; Johnsson, 1959) peuvent produire des fentes à remplissage minéral. Mais l'hypothèse de culots de glace morte doit être écartée, la région se situant à plus de $15 \mathrm{~km}$ du pied des Pyrénées. Aucun glacier n'a pu s'étendre aussi loin sur le piémont. Quant aux failles et affaissements, ils auraient atteint le dépôt sur l'ensemble de sa hauteur. Or les fentes s'amortissent en profondeur, ce qui caractérise les fissures de contraction. La dessiccation liée à des températures estivales élevées, à $2 \mathrm{~m}$ de profondeur, dans un cailloutis altéré, sous des cryoturbations, est peu plausible. Reste la gélidessiccation (Tricart et Cailleux, 1967) ou la contraction thermique, les deux sous l'influence du froid. 
Or, ici encore, toutes les fentes de froid n'impliquent pas nécessairement un pergélisol (Black, 1976; Svensson, 1977). Elles peuvent s'ouvrir, en hiver, sur plusieurs décimètres de profondeur et sur quelques millimètres de largeur. À Luquet, elles atteignent 2 à $3 \mathrm{~cm}$ de large. Elles ne résultent donc pas d'un seul hiver, mais impliquent un élargissement graduel, cumulatif, par coups de gel successifs. Si la fissuration s'est faite au taux moyen de 2 à $5 \mathrm{~mm} / \mathrm{an}$, l'ouverture des fentes aura exigé, au minimum, de 5 à 15 hivers marqués par un gel à près de $2 \mathrm{~m}$ de profondeur. Le remplissage homogène, amorphe, des fentes indique un colmatage par écoulement du lœss dans des fissures béantes, par suite de la fusion d'un mince coin de glace souterraine. Ce coin de glace aurait pris, au minimum, cinq années consécutives à se former. Si l'on définit un pergélisol comme une zone souterraine marquée par des températures inférieures à $0^{\circ} \mathrm{C}$ pendant au moins deux années consécutives, les fentes de Luquet nous renvoient ainsi à un ancien pergélisol. Nous n'en connaissons ni la durée réelle ni le moment précis de son apparition au cours du Würm.

On considère que dans des graviers, la formation des coins de glace exige des températures annuelles moyennes du sol inférieures à $-7^{\circ} \mathrm{C}$. Comme, sur le piémont pyrénéen, les cailloutis altérés baignent dans une matrice limonoargileuse, les paléotempératures souterraines auraient pû être moins rigoureuses, de l'ordre de $-2^{\circ} \mathrm{C}$ à $-4^{\circ} \mathrm{C}$ (Romanovskij, 1973). Ce qui correspondrait, selon Gold et Lachenbruch (1973), dans les années au cours desquelles s'est produit la fissuration profonde, à des températures moyennes annuelles de l'air situées entre $-3^{\circ} \mathrm{C}$ et $-10^{\circ} \mathrm{C}$.

Même en admettant des paléotempératures de $-3^{\circ} \mathrm{C}$, le climat périglaciaire, bien entendu, ne pouvait se limiter au site de Luquet. II devait couvrir le piémont des Pyrénées et, a fortiori, la montagne elle-même.

\section{LA SIGNIFICATION DES GALETS DRESSÉS}

Le lœss, les cryoturbations et les fentes en coin ne sont peut-être pas les seuls phénomènes périglaciaires présents dans les coupes de Luquet et de Nousty. Les galets dressés dans les 70 à $80 \mathrm{~cm}$ supérieurs des terrasses pourraient résulter de cycles gélivaux. Un gel saisonnier serait alors descendu, non pas à $1,10 \mathrm{~m}$ de profondeur comme l'indique la base des poches de limon, mais à 1,80 m. II n'est pas nécessairement lié aux cryoturbations. Dans la vallée d'Aspe, à $40 \mathrm{~km}$ à l'ouest des sites étudiés ici, des galets dressés au sommet de la "terrasse moyenne" d'Agnos (Gangloff et al., 1991b) sont recouverts par le lœss non cryoturbé. À Luquet, le redressement des galets semble contemporain de la mise en place de la nappe de gravats, celle-ci pouvant elle-même être d'origine périglaciaire. Comme elle est antérieure au lœss et à ses cryoturbations, nous serions en présence de deux périodes périglaciaires, superposées dans la même coupe. II y aurait eu: 1) redressement des galets et formation d'un pavage périglaciaire, après l'altération des cailloutis, 2) dépôt du lœss sur le pavage puis, 3) cryoturbation et formation des fentes de gel. Mais toutes les nappes de gravats ne sont pas nécessairement périglaciaires. Quant aux galets dressés, parce que pris dans un matériel altéré, ils pourraient résulter du réarrangement interne du cailloutis suggéré par la disparition, à Luquet, des fantômes de blocs décomposés. Ainsi, faute de données explicites, la signification paléoclimatique des arrangements de galets reste équivoque. Nous ne retiendrons des coupes, pour le moment, que les conclusions les plus probantes.

\section{CONCLUSIONS}

Au cours du Würm, entre Lourdes, Tarbes et Pau, un manteau de lœss s'est déposé sur le piémont nord des Pyrénées, notamment sur la "terrasse moyenne " et la Formation de Lannemezan. La cryoturbation würmienne a déformé ce lœss et la tranche supérieure des alluvions de terrasse. La couche active descendait à $1,10 \mathrm{~m}$ de profondeur. Elle surmontait un pergélisol soumis à des processus de fissuration, probablement par contraction thermique, donnant les deux fentes de gel de Luquet, dont l'une descend à $1,80 \mathrm{~m}$ de profondeur.

Les cryoturbations, à elles seules, nous indiquent des paléotempératures annuelles moyennes de l'air inférieures à $2^{\circ} \mathrm{C}$ et des moyennes du mois le plus froid inférieures à $-15^{\circ} \mathrm{C}$. Mais les fentes en coin montrent que, dans les faits, le paléoclimat était bien plus rigoureux. Elles impliquent un pergélisol sous des moyennes annuelles de l'air inférieures ou égales à $-3^{\circ} \mathrm{C}$. Le pergélisol, attesté par les fentes, pouvait ne toucher que le piémont pyrénéen et la montagne voisine. Son raccord éventuel (et probable) avec la zone de pergélisol continu de l'Europe du Nord-Ouest reste à être démontré.

Après la phase de cryoturbation, un sol s'est développé dans le lœss, sous une végétation steppique dont le spectre pollinique évoque la palynozone à Poaceae du Würm supérieur. Des vers de terre ont entraîné du matériel de l'horizon Ah en profondeur, dans leurs galeries. Des particules organiques ont continué de migrer, vers le bas, à travers le lœss, durant l'Holocène, comme l'indiquent les charbons de bois millimétriques, vieux d'environ trois mille ans, trouvés à la base d'une poche d'involution.

\section{REMERCIEMENTS}

Nous tenons à exprimer notre sincère gratitude aux personnes qui ont contribué à la réalisation de cette étude, dont madame Nicole Morasse pour les analyses polliniques, messieurs Paul Beauchemin et Sylvain Savoie pour les analyses sédimentologiques et monsieur Guy Frumignac pour la mise au net des figures. Messieurs Pascal Bertran, Jean-Pierre Coutard, Jean-Pierre Lautridou et Jeff Vandenberghe, par leurs suggestions et leurs critiques constructives, on contribué à améliorer la version finale du texte. Nous leur sommes particulièrement reconnaissants. Nous avons bénéficié de subventions du CRSNG (Canada), du FIR (Université du Québec à Rimouski) et du CAFIR (Université de Montréal).

\section{RÉFÉRENCES}

Alimen, H.,1964. Le Quaternaire des Pyrénées de la Bigorre. Mémoires de la Carte géologique de France, 1, $394 \mathrm{p}$ 
Andrieu, V., 1991. Dynamique du paléoenvironnement de la vallée montagnarde de la Garonne (Pyrénées centrales, France) de la fin des temps glaciaires à l'actuel. Thèse, Université de Toulouse 2, $330 \mathrm{p}$.

Birkeland, P.W., 1984. Soils and Geomorphology. Oxford University Press, $372 \mathrm{p}$.

Black, R.F., 1976. Periglacial features indicative of permafrost : Ice and soil wedges. Quaternary Research, 6 : 3-26.

Brown, J.-L. et Gangloff, P., 1980. Géliformes et sols cryiques dans le sud de l'Abitibi, Québec. Géographie physique et Quaternaire, 34(2) : 137-158.

Bülow, W.V.,1964. Ein Taschen- und Tropfenboden von Grebs (SW Mecklenburg). Geologie, 13(3) : 361-363.

Callot, G. et Juste, C., 1971. Caractérisation des sols humifères, acides, développés sur les alluvions anciennes des gaves pyrénéens. Science du Sol, $2: 5-26$.

Comité d'experts sur la prospection pédologique d'Agriculture Canada, 1987. Le système canadien de classification des sols. Seconde édition. Publication 1646. Agriculture Canada, $170 \mathrm{p}$.

Eissmann, L., 1981. Periglaziäre Prozesse und Permafroststrukturen aus sechs Kaltzeiten des Quartärs. Ein Beitrag zur Periglazialgeologie aus der Sicht des Saale-Elbe-Gebiet. Altenburger Naturwissenschaftliche Forschungen, 1, $171 p$.

Gangloff, P., Courchesne, F., Hétu, B., Jalut, G. et Richard, P.J.H., 1991a. Découverte d'un paléolac sur le piémont des Pyrénées Atlantiques (France). Zeitschrift für Geomorphologie, 35(4) : 463-478.

Gangloff P., Hétu B. et Courchesne F., 1991b. Présence d'un dépôt glaciaire sous le cailloutis de la terrasse moyenne, vallée d'Aspe (Pyrénées Atlantiques). Quaternaire, 2(3-4) : 131-133.

Gangloff, P. et Pissart, A., 1983. Évolution géomorphologique et palses minérales dans la région de Kuujjuak (Fort-Chimo). Bulletin de la Société géographique de Liège, $19:$ 119-132.

Gold, L.,W. et Lachenbruch, A., H., 1973. Thermal conditions in permafrost. A review of North American litterature, p. 3-23. In Permafrost, North American Contribution to the Second international Conference. National Academy of Science, Washington, D.C.

Gullentops, F. et Paulissen, E., 1978. The drop soil of the Eisden type. Biuletyn Peryglacjalny, 27 : 105-114.

Hétu, B. et Gangloff, P. 1989. Dépôts glaciaires du Pleistocène inférieur sur le piémont nord des Pyrénées Atlantiques. Zeitschrift für Geomorphologie, $33(4)$ : 385-403.

Heyse, I., 1983. Fossil cryoturbation types in eolian wurm late glacial sediments in Flanders (Belgium). Polarforschung, $53: 2$.

Hubschman, J., Icole, M., Jalut, G., Revel, J.-C., Taillefer F. et Bourgeat, F., 1980. Le Quaternaire nord pyrénéen. In Problèmes de stratigraphie quaternaire en France et dans les pays limitrophes. Supplément du Bulletin de l'Association trançaise pour l'ètude du Quaternaire, N.S. 1 : 307-313.

Icole, M., 1971. Accumulation d'halloysite dans les paléosols des terrasses mindéliennes et du Quaternaire ancien des Pyrénées nord-occidentales. Comptes rendus de l'Académie des Sciences de Paris, 272-D : 1216-1218.
- 1974. Géochimie des altérations dans les nappes d'alluvions du piémont occidental nord-pyrénéen. Éléments de paléopédologie quaternaire. Sciences géologiques, $40,200 p$.

Jalut, G., 1984. L'action de l'homme sur la forêt montagnarde des Pyrénées ariégeoises et orientales depuis 4000 ans d'après l'analyse pollinique. Actes $106^{\circ}$ Congrès international des Sociétés savantes., Perpignan 1981, CTHS, Paris, p. 163-172.

Johnsson, G., 1959. True and false ice-wedges in southern Sweden. Geografiska Annaler, $41: 15-33$.

Kaiser, K.,1960. Klimazeugen des periglazialen Dauerfrostbodens in Mittelund West-Europa. Eiszeitalter und Gegenwart, $11:$ 121-141.

Maarleveld, G.-C., 1976. Periglacial phenomena and the mean annual temperature during the last glacial time in the Netherlands. Biuletyn Peryglacjalny, 26 : 57-78.

Pissart, A., 1982. Déformations de cylindres de limon entourés de graviers sous l'action d'alternances gel/dégel. Biuletyn Peryglacjalny, 29 : 219-229.

1987. Géomorpohologie périglaciaire. Rijksuniversiteit Gent, 135 p.

Pissart, A. et Gangloff, P., 1984. Les palses minérales et organiques de la vallée de l'Aveneau près de Kuujjuak (Fort-Chimo), Québec sub-arctique. Géographie physique et Quaternaire, $38(3): 217-228$.

Poser, H., 1948. Boden- und Kilmaverhältnisse in Mittel- und Westeuropa während der Würmeiszeit. Erdkunde, 2 : 53-68.

Reille, M., 1990. Leçons de palynologie et d'analyse pollinique. Éditions du CNRS, Paris, $206 \mathrm{p}$.

Romanovskij, N.N., 1973. Regularities in formation of frost fissures and development of frost fissure polygons. Biuletyn Peryglacjalny, 23 : 237-277.

Svensson, H., 1977. Observations on polygonal fissuring in non-permafrost areas of the Norden Countries. In H. Poser, édit., Formen, Formengesellschaften und Untergrenzen in den heutigen periglazialen Hochstufen der Hochgebirge Europas und Afrikas zwischen Arktis und Äquator. Abhandlungen der Akademie der Wissenschaften in Göttingen, Mathematisch- Physikalische Klasse, $31:$ 63-76.

Texier, J.-P. et Bertran, P., 1993. Données nouvelles sur la présence d'un pergélisol en Aquitaine au cours des dernières glaciations. Permafrost and Periglacial Processes, 4(3) : 183-198.

Tricart, J. et Cailleux, A., 1967. Le modelé des régions périglaciaires. Traité de géomorphologie. tome 2. SEDES, Paris, $512 \mathrm{p}$.

Vandenberghe, J. et Pissart, A., 1993. Permafrost changes in Europe during the last glacial. Permafrost and Periglacial Processes, 4(2) : 121-135.

Vandenberghe, J. et Van de Broeck, P., 1982. Weichselian convolution phenomena and processes in fine sediments. Boreas, 11(2) : 299-315.

Velitchko, A.A.,1982. Paleogeography of Europe during the last one hundred thousand years. Nauka, Moscou, $156 \mathrm{p}$.

Washburn, A.L., 1973. Periglacial processes and environment. Edward Arnold, $320 \mathrm{p}$. 